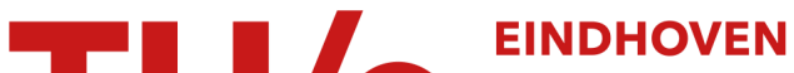 \\ UNIVERSITY OF \\ TECHNOLOGY
}

\section{Route towards huge magnetoresistance in doped polymers}

Citation for published version (APA):

Kersten, S. P., Meskers, S. C. J., \& Bobbert, P. A. (2012). Route towards huge magnetoresistance in doped polymers. Physical Review B, 86, 1-6. [045210]. https://doi.org/10.1103/PhysRevB.86.045210

DOI:

10.1103/PhysRevB.86.045210

Document status and date:

Published: 01/01/2012

\section{Document Version:}

Publisher's PDF, also known as Version of Record (includes final page, issue and volume numbers)

\section{Please check the document version of this publication:}

- A submitted manuscript is the version of the article upon submission and before peer-review. There can be important differences between the submitted version and the official published version of record. People interested in the research are advised to contact the author for the final version of the publication, or visit the $\mathrm{DOI}$ to the publisher's website.

- The final author version and the galley proof are versions of the publication after peer review.

- The final published version features the final layout of the paper including the volume, issue and page numbers.

Link to publication

\section{General rights}

Copyright and moral rights for the publications made accessible in the public portal are retained by the authors and/or other copyright owners and it is a condition of accessing publications that users recognise and abide by the legal requirements associated with these rights.

- Users may download and print one copy of any publication from the public portal for the purpose of private study or research.

- You may not further distribute the material or use it for any profit-making activity or commercial gain

- You may freely distribute the URL identifying the publication in the public portal.

If the publication is distributed under the terms of Article 25fa of the Dutch Copyright Act, indicated by the "Taverne" license above, please follow below link for the End User Agreement:

www.tue.nl/taverne

Take down policy

If you believe that this document breaches copyright please contact us at:

openaccess@tue.nl

providing details and we will investigate your claim. 


\title{
Route towards huge magnetoresistance in doped polymers
}

\author{
S. P. Kersten, ${ }^{1}$ S. C. J. Meskers, ${ }^{2}$ and P. A. Bobbert ${ }^{1}$ \\ ${ }^{1}$ Department of Applied Physics, Eindhoven University of Technology, P.O. Box 513, 5600 MB Eindhoven, The Netherlands \\ ${ }^{2}$ Department of Chemical Engineering and Chemistry, Eindhoven University of Technology, P.O. Box 513, \\ 5600 MB Eindhoven, The Netherlands
}

(Received 31 January 2012; published 20 July 2012)

\begin{abstract}
Room-temperature magnetoresistance of the order of $10 \%$ has been observed in organic semiconductors. We predict that even larger magnetoresistance can be realized in suitably synthesized doped conjugated polymers. In such polymers, ionization of dopants creates free charges that recombine with a rate governed by a competition between an applied magnetic field and random hyperfine fields. This leads to a spin-blocking effect that depends on the magnetic field. We show that the combined effects of spin blocking and charge blocking, the fact that two free charges cannot occupy the same site, lead to a magnetoresistance of almost two orders of magnitude. This magnetoresistance occurs even at vanishing electric field and is therefore a quasiequilibrium effect. The influences of the dopant strength, energetic disorder, and interchain hopping are investigated. We find that the dopant strength and energetic disorder have only little influence on the magnetoresistance. Interchain hopping strongly decreases the magnetoresistance because it can lift spin-blocking and charge-blocking configurations that occur in strictly one-dimensional transport. We provide suggestions for realization of polymers that should show this magnetoresistance.
\end{abstract}

DOI: 10.1103/PhysRevB.86.045210

PACS number(s): 72.80.Le, 72.20.My, 73.50.-h, 75.47.-m

\section{INTRODUCTION}

In the last decade, large room-temperature magnetic field effects in the current and electroluminescence of devices of organic semiconductors have been found. ${ }^{1-10}$ The precise mechanisms behind these effects are still debated, but agreement is arising that the effects are caused by the magnetic-field sensitivity of spin-selective reactions between spin-carrying electronic excitations (electrons, holes, triplet excitons). Striking analogies exist with mechanisms known in the field of spin chemistry. ${ }^{11}$ The involvement of hyperfine fields has recently been demonstrated by the occurrence of isotope effects. ${ }^{9,10}$ Isotope substitution in organic semiconductors (e.g., the replacement of hydrogen by deuterium or ${ }^{12} \mathrm{C}$ by ${ }^{13} \mathrm{C}$ ) leads to different nuclear magnetic moments and therefore to a different hyperfine interaction between the nuclei and spin-carrying excitations, while leaving all other electronic properties of the semiconductor unchanged. It has been shown that the magnetic field dependence scales accordingly., ${ }^{9}, 10$

The electronic spin in an organic semiconductor interacts with many (typically of the order of ten or more) nuclear spins. As a consequence, this interaction can be quite well described by assuming that the electronic spin experiences a classical, quasistatic, and random hyperfine field, having a Gaussian distribution with a standard deviation $B_{\mathrm{hf}}$ of the order of a milli-Tesla. ${ }^{12,13}$ The evolution of the spin state of a pair of spin-carrying excitations is then determined by the sum of an externally applied magnetic field $B$ and the local hyperfine fields, which are different for the two excitations. If the reaction between the two excitations is spin selective, the reaction rate changes when the magnitude of $B$ surpasses $B_{\mathrm{hf}}$, giving rise to a $B$-dependent reaction rate. Magnetic field effects in the current of unipolar organic devices have been explained by a mechanism in which two electrons or holes react to form a singlet bipolaron. ${ }^{14}$ Magnetic field effects in the electroluminescence of bipolar devices have been explained by a mechanism in which electrons and holes react to form singlet or triplet excitons with different rates. ${ }^{9,10,15}$ Spin-selective reactions between electrons and holes as well as between electrons or holes and triplet excitons have also been suggested to be responsible for magnetic field effects in the current. ${ }^{3-5}$

A magnetic field effect in the current is usually interpreted as a magnetoresistance. The reported magnetoresistance of present organic devices is of the order of $10 \%$ at rather high electric fields. ${ }^{16}$ With the insight that magnetic field effects in organic semiconductors are caused by spin-selective reactions between spin-carrying excitations, one may ask the question if it is possible to design organic materials with even higher magnetoresistance at a low electric field. The manufacturing of an organic material with very large magnetoresistance at low magnetic and electric field, to be used in highly sensitive magnetic sensors or maybe even magnetic switches, would be of large technological interest. Such sensors could be integrated with other cheap and flexible organic electronics. The present paper is concerned with a theoretical survey of this possibility.

The route we propose towards high magnetoresistance at low electric field is the use of doped $\pi$-conjugated polymers with specific properties. Doped polymers have been investigated in great detail because of their conducting properties. In these polymers free charges created by ionization of dopants move along the polymer chains. Our present interest is in the concurrent creation of free spins, carried by the free charges and the ionized dopants. We will consider the case of intrinsically doped polymers where the dopants are part of the polymer chains themselves. To obtain a large magnetoresistance it is important that the coupling between the monomeric units of the polymers is small, such that the hopping transport between these units takes place with a rate that is smaller than the hyperfine precession rate. This makes the proposed polymers different from commonly used conjugated polymers, where charges can delocalize over several monomers. We propose to achieve the required 
small hopping rate by inserting spacer units in between the monomers. Under this condition, the recombination rate of a free charge with an ionized dopant will become magnetic field dependent, leading to magnetoresistance. Another important condition for obtaining large magnetoresistance is that the charge transport is one dimensional. In this case, free charges contributing to transport are forced to regularly recombine with the ionized dopants. In principle, polymers are ideal in this respect, because charge transport mainly takes place along the polymer chain. However, interchain hopping can also take place, and this may allow a free charge to hop around a dopant. In order to obtain a large magnetoresistance, interchain hopping will therefore have to be suppressed. Suppression of interchain hopping can be achieved by introducing side groups.

This paper is built up as follows. In Sec. II we will introduce our model system for a doped polymer. We will show that in the case of low density of free charges in the system the problem of finding its conductivity can be mapped onto that of a resistor model. The latter problem can be easily solved. In this case, the magnetoresistance is solely caused by a spin-blocking effect in the recombination of a free charge with a dopant. In the general case of high free electron density we find the conductivity from Monte Carlo simulations. In these simulations the effect of charge blocking is included, i.e., the effect that two free charges are not allowed to occupy the same site due to their Coulomb repulsion. In Sec. III we present the results of the resistor model and those of the Monte Carlo simulations. The influences of the dopant strength, energetic disorder, and interchain hopping are investigated. In Sec. IV we discuss how the envisaged doped polymers could be realized. Section V contains a summary and the main conclusions.

\section{MODEL}

We model a polymer chain as a sequence of sites along which nearest-neighbor hopping of localized charges occurs; see Fig. 1. For simplicity, we assume that dopant sites are distributed periodically within the chain, with a period of $n$ sites. Because of the one-dimensionality, the case of arbitrarily distributed dopants follows from combining the results of periodically doped chains with appropriate weights for different $n$. We consider the case of donors (the case of acceptors is completely equivalent) with a highest occupied molecular orbital (HOMO) that is energetically separated by a small energy $\Delta$ from the lowest unoccupied molecular orbital (LUMO) of the host sites. In that case, ionization of a donor can take place at thermal conditions by hopping of an electron

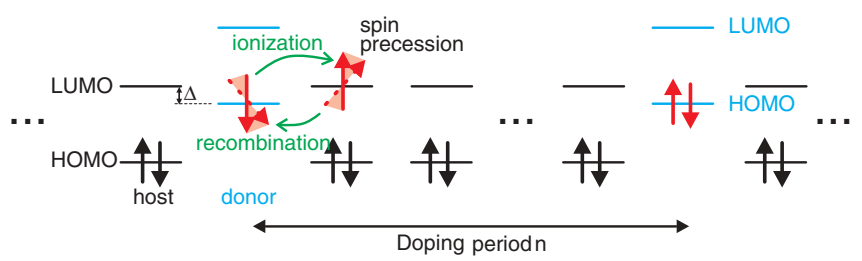

FIG. 1. (Color online) Doped polymer chain containing donor sites with period $n$. A small energy difference $\Delta$ between donor HOMO and host LUMO allows ionization of donors, followed by precession of the donor spin and the free-electron spin (red arrows). Recombination occurs back to the spin-singlet unionized donor state. from the donor to a neighboring host site. With the unionized donor being a spin singlet, the combination of the free electron and the ionized donor, both having spin $\frac{1}{2}$, will initially also be a spin singlet. However, the different hyperfine fields at the positions of the free electron and ionized donor mixes in triplet character, which reduces the recombination rate back to the singlet state of the unionized donor. Spin mixing also changes the recombination rate of the free electron with other ionized donors. The spins of the free electron and the ionized donor might happen to be in a triplet configuration, for which recombination would not be allowed. Spin mixing by the random hyperfine fields will then raise the recombination rate by mixing in singlet character. An applied magnetic field suppresses the spin mixing and hence the recombination. In both cases, a magnetic field-dependent spin blocking occurs that leads to magnetoresistance, even at vanishing electric field.

The combination of incoherent spin-selective hopping and coherent spin evolution can be described with the stochastic Liouville equation. ${ }^{17,18}$ It follows from this equation that when the hopping rate $k_{\text {hop }}$ is much larger than the hyperfine precession frequency $\omega_{\mathrm{hf}}$ of typically $10^{8} \mathrm{~s}^{-1}\left(\omega_{\mathrm{hf}}=\gamma B_{\mathrm{hf}}\right.$, with $\gamma$ the gyromagnetic ratio) the effects of the hyperfine fields will be quenched and no magnetoresistance occurs. The largest magnetoresistance occurs when $k_{\text {hop }}$ is much smaller than $\omega_{\mathrm{hf}}$, and this "slow-hopping" case is the case we consider from now on. The observation of large magnetic field effects in organic semiconductors ${ }^{1-10}$ indicates that the hopping rate can indeed be smaller than or at least comparable to the hyperfine precession frequency. Because coherent effects between eigenstates of the spin Hamiltonian vanish in the limit of slow hopping, we only need to consider the occupancy of the (localized) spin eigenstates and hopping between these states. $^{14}$

\section{A. Resistor model}

In Fig. 2(a) we consider a part of the chain for the simplest situation, with periodically a donor and a host site, corresponding to a period $n=2$ in Fig. 1 . To demonstrate the essence of the magnetoresistance occurring in this system, we take $\Delta=0$ and first consider what we will call a "low electron density," corresponding to at most one free electron on the chain. In this case, there is either only one donor unionized or all donors are ionized and there is one free electron on a host site. In the donor-host-donor sequence of Fig. 2(a) an extra electron can be on one of the three sites $i=1,2,3$. If the electron is on either of the donor sites $(i=1,3)$, that donor is unionized, while the other is ionized. The spin eigenstates then correspond to a spin at the ionized donor that can be either parallel (P) or antiparallel (A) to the total effective magnetic field $\mathbf{B}_{\text {tot }, i}$ at that donor, which is the sum of the external magnetic field $\mathbf{B}$ and the local random hyperfine field $\mathbf{B}_{\mathrm{hf}, i}$. We label these eigenstates as $--\mathrm{P},--\mathrm{A}, \mathrm{P}--$, and $\mathrm{A}--$. If the electron is on the host site $i=2$, there are spins at all three sites and the corresponding eigenstates are labeled as PPP, AAA, APP, PAA, PAP, APA, PPA, and AAP. In Fig. 2(a) two consecutive hops are indicated, corresponding to $--\mathrm{P} \rightarrow \mathrm{PAP} \rightarrow \mathrm{P}--$.

In the presence of a vanishingly small electric field $F$ the current through the hopping network can be calculated from a 

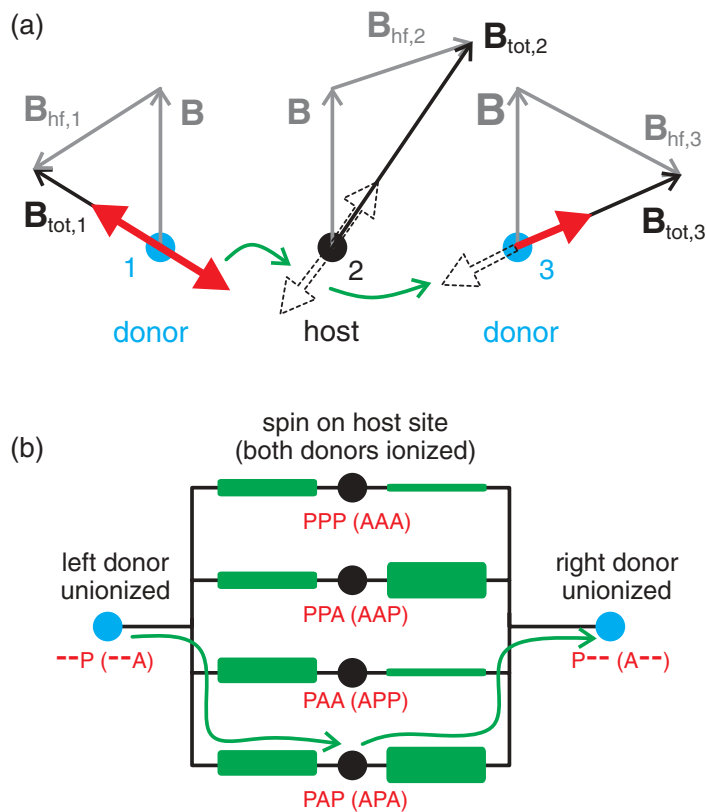

FIG. 2. (Color online) (a) Donor-host-donor sequence $i=1,2,3$ for the case $n=2$. The spin eigenstates are indicated by the red and dashed arrows. The total effective magnetic fields $\mathbf{B}_{\mathrm{tot}, i}$ are the sums of the external field $\mathbf{B}$ and local random hyperfine fields $\mathbf{B}_{\mathrm{hf}, i}$. Two possible consecutive hopping events are shown, corresponding to ionization at the left donor and recombination at the right donor. (b) Resistor network corresponding to hopping between the spin eigenstates of (a), with conductances indicated by the thickness of the drawn resistors. The arrows show the current flow corresponding to the two hopping events in (a). The labeling of the states is explained in the main text.

mapping onto a resistor network, ${ }^{19}$ where the above eigenstates correspond to the nodes of the network. Generalizing the result of Ref. 19, the conductance between nodes $p$ and $q$ is given by

$$
\tilde{G}_{p q}=\frac{e^{2} \tilde{k}_{p q}^{\mathrm{symm}}}{k_{\mathrm{B}} T} \exp \left(\frac{E_{\mathrm{F}}}{k_{\mathrm{B}} T}-\frac{E_{p}+E_{q}}{2 k_{\mathrm{B}} T}\right),
$$

where $k_{\mathrm{B}} T$ is the thermal energy and $e$ the electronic charge. $E_{p}$ and $E_{q}$ are the energies associated with nodes $p$ and $q$ of the network, and $E_{\mathrm{F}}$ is the Fermi energy, which determines the number of extra charges on the chain (in this case one). $\tilde{k}_{p q}^{\text {symm }}$ is a symmetrized hopping rate: $\tilde{k}_{p q}^{\text {symm }} \equiv\left(\tilde{k}_{p q} \tilde{k}_{q p}\right)^{1 / 2}$. The rates $\tilde{k}_{p q}$ are equal to a spin-independent rate $k_{p q}$ multiplied by a spin-projection factor $P_{p q}: \tilde{k}_{p q} \equiv P_{p q} k_{p q}$. For hops of the electron between two host sites we have $P_{p q}=\cos ^{2}\left(\theta_{p q} / 2\right)$ $\left[\sin ^{2}\left(\theta_{p q} / 2\right)\right]$ for hops between eigenstates for which the spin keeps (changes) its orientation with respect to the direction of the local effective magnetic field, where $\theta_{p q}$ is the angle between the effective magnetic fields of the two sites. For recombination from or ionization to a state for which the dopant spin is parallel and the electron spin antiparallel to the local effective magnetic field, or vice versa, we have $P_{p q}=$ $\frac{1}{2} \cos ^{2}\left(\theta_{p q} / 2\right)$. If both spins are parallel or antiparallel to the local effective magnetic field we have $P_{p q}=\frac{1}{2} \sin ^{2}\left(\theta_{p q} / 2\right)$. The latter factors are the projections onto the spin-singlet subspace. The resistor network corresponding to Fig. 2(a) for the case $\Delta=0, n=2$, and $k_{p q}=k_{\text {hop }}$ for all allowed hops is given in Fig. 2(b), where the thickness of the drawn resistors indicates their conductance. We note that reversing all spins leaves the network unchanged, which is indicated by the labels between brackets. It is straightforward to set up resistor networks for larger values of $n$, but their complexity increases rapidly with increasing $n$.

The charge-carrier mobility can be obtained from the resistance $R(B)$ of a chain of $N \gg 1$ sites by $\mu(B)=$ $\exp \left(-E_{\mathrm{F}} / k_{\mathrm{B}} T\right) N a^{2} / e R(B)$, where $a$ is the intersite distance. The resistance $R(B)$ is the sum of the resistances of many of the above resistor networks with random hyperfine fields and is obtained by a hyperfine-field average of the resistance of such a network. For simplicity we assume equal standard deviations $B_{\mathrm{hf}}$ for the Gaussian distributions of the donor and host hyperfine fields, and equal gyromagnetic ratios. If donor and acceptor have different $B_{\mathrm{hf}}$ this will only change the results for nonzero finite $B$ and not the magnetoresistance.

\section{B. Monte-Carlo simulations}

The resistor model does not take into account Coulomb interactions between the free electrons, and it is therefore limited to the case of low electron densities. The most important effect of Coulomb interactions in organic semiconductors is to prevent the presence of two charges on the same site. ${ }^{20} \mathrm{We}$ take this effect into account in Monte Carlo (MC) simulations that we performed for long chains. In these simulations hops are chosen randomly with weights proportional to the rates $\tilde{k}_{p q}$ discussed above, where now two free electrons are not allowed to occupy the same site. After each hop the time is increased with a random time step drawn from an exponential distribution with a decay time equal to the inverse of the sum of the rates of all possible hops. A small electric field $F$ is applied that leads to a net drift of the electrons along the chain by decreasing the rate of the up-field hops by a factor $\exp \left(-e a F / k_{\mathrm{B}} T\right)$. The mobility is obtained from the average electron drift velocity $v(B)$ by $\mu(B)=v(B) / F$. We took $F$ small enough to be in the regime where $v(B)$ is linear in $F$, yet large enough to obtain a sufficient accuracy in $\mu(B)$.

We considered the charge-neutral case of "high electron density," where the number of free electrons is equal to the number of ionized donors. The typical chain lengths that we took were $2 \times 10^{5}(n=2)$ up to $3 \times 10^{6}(n=30)$ sites. Steady-state situations were obtained after $2 \times 10^{9}$ hops, after which $v(B)$ was calculated for $2 \times 10^{9}(n=2)$ up to $3 \times 10^{10}$ $(n=30)$ hops. Averages were taken over $10(n=30)$ up to $200(n=2)$ hyperfine-field configurations.

MC simulations were also performed for the case of low electron density. In that case the mobility can alternatively be obtained by using Einstein's relation $\mu(B)=e D(B) / k_{\mathrm{B}} T$, and calculating the diffusion constant $D(B)$ from MC simulations of diffusion of a single electron along a chain in the absence of an electric field. The chain lengths that we took were the same as for the case of high electron density. The number of hops in the calculation of the diffusion constant were $2 \times 10^{6}(n=30)$ up to $8 \times 10^{6}(n=2)$. Averages were taken over $10^{3}(n=10)$ up to $3.5 \times 10^{3}(n=2)$ hyperfine-field configurations. 


\section{RESULTS}

\section{A. Low electron density}

We start by considering the case of low electron density, where there is at most one free electron on the chain. The results for the mobility obtained from the resistor model are shown in Fig. 3(a). In the limit $B \rightarrow \infty$ we obtain $\mu(\infty)=\left[n^{2} /(4 n-3)(n+2)\right] e k_{\mathrm{hop}} a^{2} / k_{\mathrm{B}} T$ (crosses). Results for $\mu(0)$ were evaluated for networks up to $n=5$ (pluses). For $n \rightarrow \infty$ and finite $B$ the effect of the presence of the donors vanishes and we thus obtain $\mu(0)=e k_{\mathrm{hop}} a^{2} / k_{\mathrm{B}} T$. The results for the mobility using the Einstein relation, with the diffusion constant obtained from MC simulations, are also displayed ( $B=0$ : filled circles; $B \rightarrow \infty$ : open circles). The agreement with the results of the resistor model is perfect. These results demonstrate explicitly that the magnetic field effect occurs even in the absence of an electric field.

The magnetic field effect in the mobility, defined as MFE $\equiv$ $\operatorname{MFE}(\infty)$, where $\operatorname{MFE}(B) \equiv[\mu(B)-\mu(0)] / \mu(0)$, is shown in Fig. 3(b) (half-filled circles). For $n=2$ we obtain a magnetic field effect of $-37 \%$, which grows to $-75 \%$ in the limit $n \rightarrow$ $\infty$. The physical reason for the magnetic field effect is the spin blocking that occurs in the case of large $B$ due to the occurrence of spin configurations where the spin of the electron has the same orientation as that of a neighboring ionized donor site, preventing recombination and further transport. The reason why the magnetic field effect increases for increasing doping period $n$ is that spin blocking then becomes more effective. At

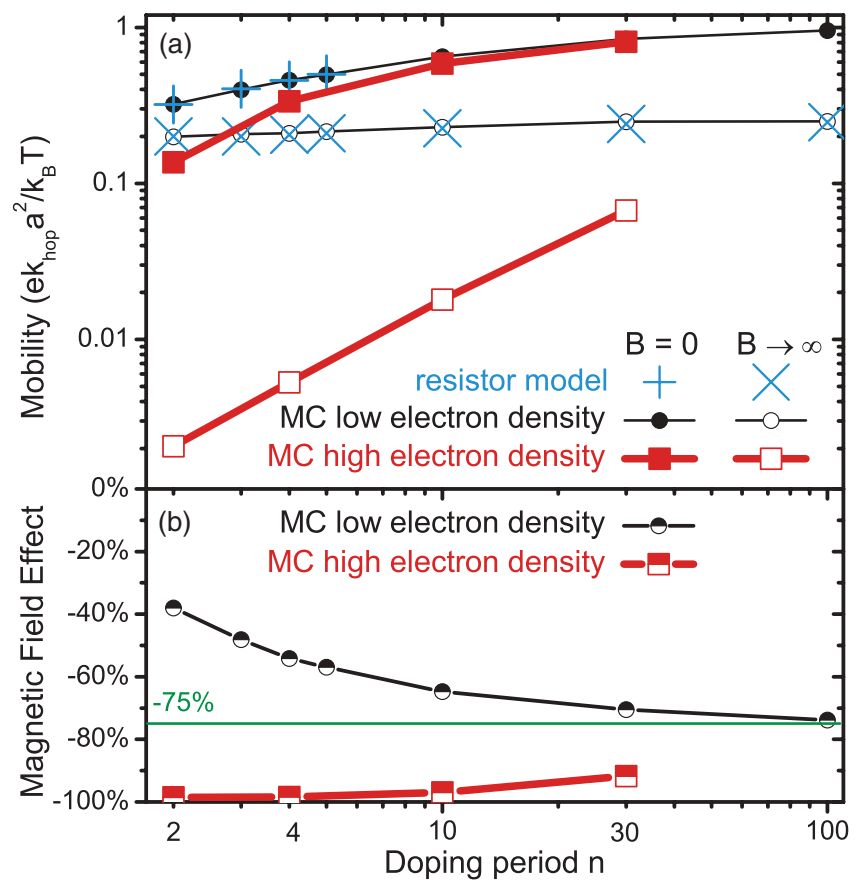

FIG. 3. (Color online) (a) Mobility as a function of doping period $n$ for $B=0$ and $B \rightarrow \infty$, obtained from the resistor model (plusses and crosses) and from Monte-Carlo (MC) simulations for low (closed and open circles) and high (closed and open squares) electron density (see main text for the definition of high and low). (b) Magnetic field effect in the mobility. The horizontal line shows the asymptotic magnetic field effect of $-75 \%$ in the absence of interaction. The error in the MC results is smaller than or equal to the size of the symbols. short doping period a spin-blocking situation of a free charge and an ionized donor in a configuration with parallel spins can be lifted not only by spin mixing by hyperfine fields, but also by return of the free charge to the ionized donor that was last visited. Recombination at this donor and subsequent re-ionization of this donor randomizes the spin of the free charge, which can lift the spin blocking. This process becomes more unlikely for a longer doping period.

We checked that in the MC simulations at zero electric field the equilibrium occupancies of all states are equal. This should be the case because the hyperfine energy scale of $\mu \mathrm{eV}$ of energy differences between the states is orders of magnitude smaller than the thermal energy scale of $10 \mathrm{meV}$. A magnetic field of the order of the hyperfine fields therefore cannot lead a change of equilibrium occupancies. We note that existing explanations of magnetic field effects involve driven reactions between spin-carrying excitations and therefore assume a nonequilibrium situation. By contrast, the present magnetoresistance is a quasiequilibrium effect.

\section{B. High electron density}

We now consider the case of high electron density, where the number of free electrons is equal to the number of ionized donors and the chain is electrically neutral. The results for the mobility and the magnetic field effect in the mobility as obtained from MC simulations for this case are given by the squares in Fig. 3. For large doping period $n$ the results approach the case of low electron density, as expected. Contrary to that case, however, we now see that the size of the magnetic field effect increases with decreasing $n$. For $n=2$ a magnetic field effect of $-98.5 \pm 0.3 \%$ is found, corresponding to a magnetoresistance of almost two orders of magnitude. Figure 3(a) shows that the main cause for this huge magnetoresistance is a dramatic drop in the mobility for $B \rightarrow \infty$ at low doping period. The reason for this huge magnetoresistance is that the spin blocking is now enhanced by charge blocking: since two electrons cannot occupy the same site, a single spin-blocked electron blocks all electrons that would otherwise be able to pass it.

\section{Line shapes}

Figure 4 shows $\operatorname{MFE}(B)$ for several doping periods, for low (circles) and high (squares) electron density. As fitting functions for the line shapes we use a Lorentzian $\left(B^{2} /\left[B^{2}+\right.\right.$ $\left.B_{0}^{2}\right]$, solid line) or a non-Lorentzian $\left(B^{2} /\left[|B|+B_{0}\right]^{2}\right.$, dashed line). In studies of magnetoresistance in devices with different organic semiconductors these two line shapes were observed. ${ }^{16}$ For low electron density, the line shape is Lorentzian for small $n$, but changes to the non-Lorentzian between $n=10$ and 30. For high electron density, the line shapes are always nonLorentzian and broader than for low electron density.

\section{Influences of the dopant strength and energetic disorder}

We investigated the influences of the donor-host HOMOLUMO energy offset $\Delta$, which is a measure of the dopant strength of the donor, and of energetic disorder in the site energies of donor and host. For simplicity we consider only 


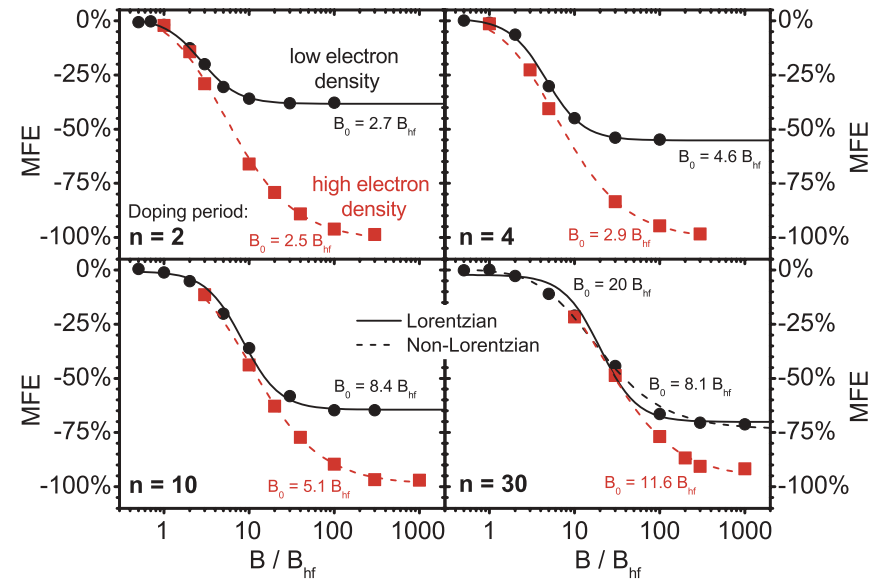

FIG. 4. (Color online) Line shapes of $\operatorname{MFE}(B)$ at low (circles) and high (squares) electron density, for four different doping periods. Lorentzian $\left(B^{2} /\left[B^{2}+B_{0}^{2}\right]\right.$, solid line) and non-Lorentzian $\left(B^{2} /\left[|B|+B_{0}\right]^{2}\right.$, dashed line) fits are shown with the corresponding values of $B_{0}$.

the case of low electron density. We expect that the conclusions drawn will qualitatively also hold for high electron density.

In the resistor model, the effect of a finite $\Delta$ manifests itself in a difference between the spin-independent part of the conductance between two host sites, $G_{\text {host }}$, and the conductance between a donor and a host site, $G_{\text {donor }}$. We note that the relevant dimensionless parameter is $\Delta / k_{\mathrm{B}} T$. Since for the case of a doping period $n=2$ there are only hops between donor and host sites, all hops are affected equally by a change in $\Delta$ and there is thus no dependence of the magnetic field effect on $\Delta$ for this case. For doping periods $n=3,4$, and 5 , the magnetic field effect in the mobility as a function of the ratio $G_{\text {host }} / G_{\text {donor }}$ is shown in Fig. 5. The results shown for low electron density in Fig. 3 correspond to $G_{\text {host }}=G_{\text {donor }}$ and lie on the dashed line in Fig. 5. We see in Fig. 5 that there is a gradual decrease in the magnitude

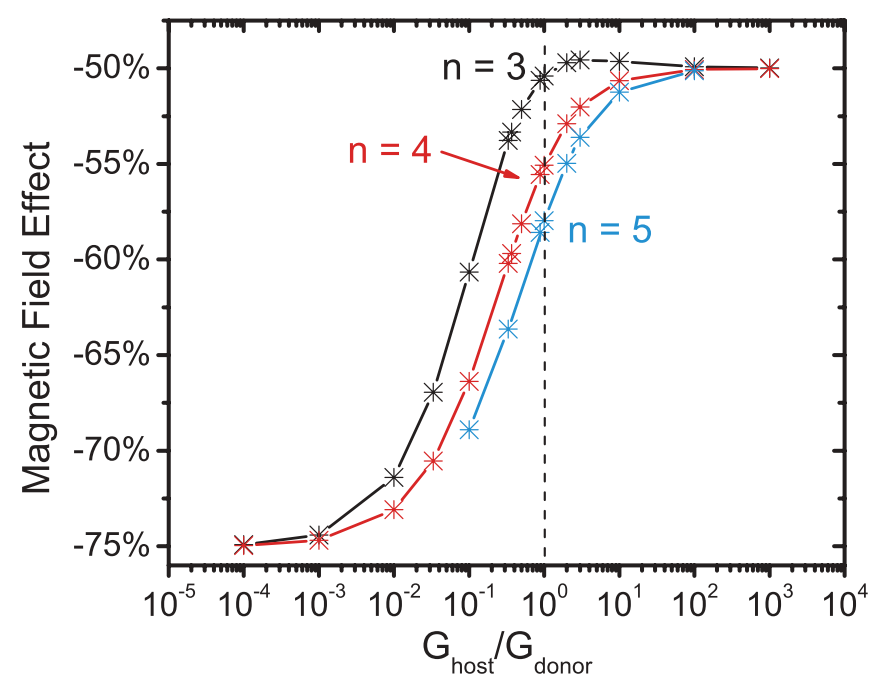

FIG. 5. (Color online) Magnetic field effect in the mobility at low electron density as a function of the ratio between $G_{\text {host }}$ and $G_{\text {donor }}$, for doping periods $n=3,4$, and 5. Points lying on the dashed line correspond to the data shown in Fig. 3. of the magnetic field effect from $75 \%$ when $G_{\text {host }} \ll G_{\text {donor }}$ to $50 \%$ when $G_{\text {host }} \gg G_{\text {donor }}$. Except for a small region in $G_{\text {host }} / G_{\text {donor }}$ for $n=3$, the magnitude of the magnetic field effect never gets smaller than $50 \%$.

The dependence of $G_{\text {host }} / G_{\text {donor }}$ on $\Delta$ is determined by the specific hopping model. In modeling studies of charge transport in organic semiconductors usually Miller-Abrahams ${ }^{21}$ or Marcus ${ }^{22}$ hopping models are used. For Miller-Abrahams hopping we find:

$$
\frac{G_{\text {host }}}{G_{\text {donor }}}=\left\{\begin{array}{lll}
\exp \left(-\Delta / k_{\mathrm{B}} T\right) & \text { if } & \Delta \leqslant 0 \\
1 & \text { if } & \Delta>0
\end{array},\right.
$$

while for Marcus hopping we find:

$$
\frac{G_{\text {host }}}{G_{\text {donor }}}=\exp \left(\frac{-\Delta+\Delta^{2} / 2 E_{\mathrm{r}}}{2 k_{\mathrm{B}} T}\right) .
$$

Here, $E_{\mathrm{r}}$ is the reorganization energy, which is typically of the order of $0.1 \mathrm{eV}$ (approximately $4 k_{\mathrm{B}} T$ at room temperature). ${ }^{23}$

Figure 6 shows the magnetic field effect in the mobility as a function of the offset $\Delta$ for doping period $n=4$, for both (a) Miller-Abrahams and (b) Marcus hopping. It is clear from Fig. 6 that the type of hopping determines the effect of $\Delta$ on the magnetic field effect. While for Miller-Abrahams hopping the magnetic field effect is constant for $\Delta>0$, for Marcus hopping the magnitude of the effect can both decrease or increase as a function of $\Delta$ for realistic $E_{\mathrm{r}}$. For Miller-Abrahams hopping, we also calculated the magnetic field effect using MC simulations to confirm the results of the resistor model. The agreement is perfect; see the squares in Fig. 6(a).

We also studied the effect of energetic disorder, again for the case of low electron density. We took Gaussian energetic disorder with a standard deviation $\sigma$ both for the HOMO energies of the donor sites and the LUMO energies of the host sites. The relevant dimensionless parameter is $\sigma / k_{\mathrm{B}} T$.

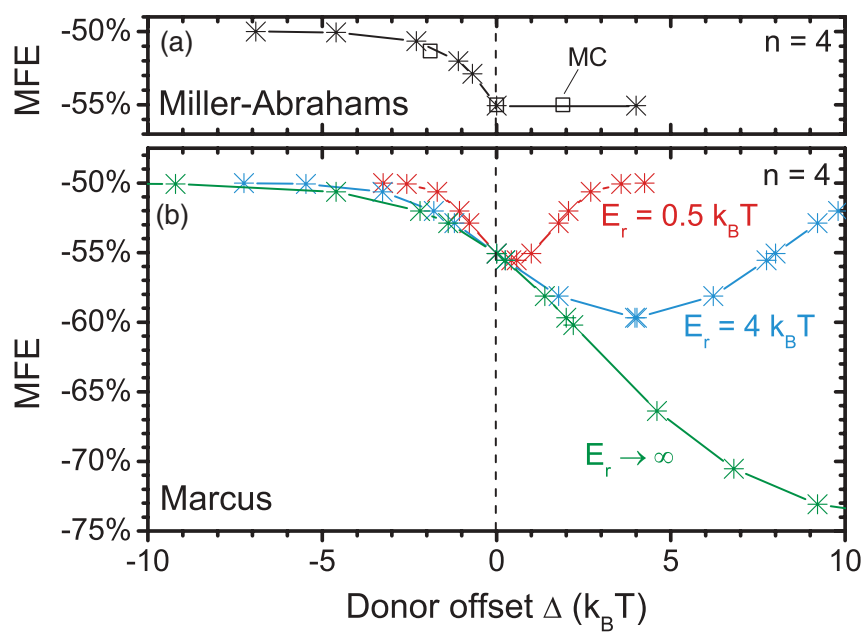

FIG. 6. (Color online) Magnetic field effect (MFE) in the mobility at low electron density as a function of energy offset $\Delta$ for $n=4$, for (a) Miller-Abrahams hopping and (b) Marcus hopping. The asterisks are calculated with the resistor model while the squares are obtained with MC simulations. In the case of Marcus hopping, results are shown for three values of the reorganization energy. The dashed line corresponds to the case considered in Fig. 3. 


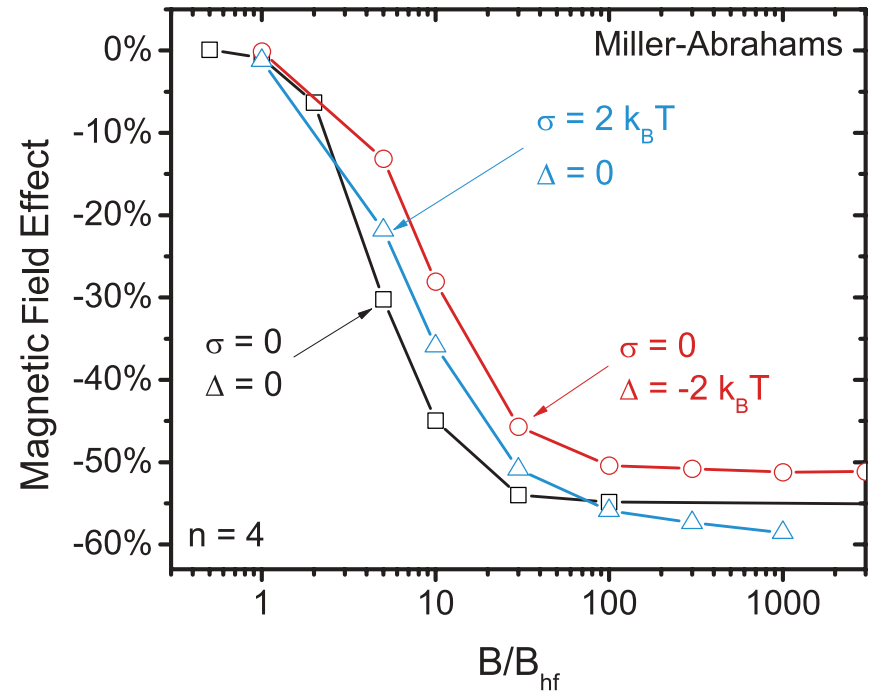

FIG. 7. (Color online) $\operatorname{MFE}(B)$ found from MC simulations at low electron density for $n=4$ and different combinations of the energy offset $\Delta$ and strength of the energetic disorder $\sigma$.

For the special case $n=2$ there are two types of conductances within each donor-host-donor sequence: the ones between the middle host site and the left donor site, and the ones between the middle host site and the right donor site. A change in the ratio between the spin-independent parts of those two types of conductances with respect to unity increases the magnitude of the magnetic field effect in the mobility from $37 \%$ at a ratio of unity (the case considered in Sec. III A) to $50 \%$ at an infinite or zero ratio. For $n>2$, a difference between the energies of neighboring host sites decreases $G_{\text {host }}$, while an energy difference between a host and a donor site can both increase or decrease $G_{\text {donor }}$, depending on $\Delta$ and, in the case of Marcus hopping, $E_{\mathrm{r}}$. Figure 7 compares $\operatorname{MFE}(B)$ when $\sigma=\Delta=0$ (the case considered in Sec. III A) to the case $\Delta=-2 k_{\mathrm{B}} T$ and $\sigma=0$, and to the case $\Delta=0$ and $\sigma=2 k_{\mathrm{B}} T$, for Miller-Abrahams hopping. The results in Fig. 7 were obtained from MC simulations. The case of positive $\Delta$ is identical to that of $\Delta=0$ for Miller-Abrahams hopping; see Fig. 6(a). It is clear from Fig. 7 that neither nonzero $\Delta$ nor nonzero $\sigma$ changes the magnetic field effect significantly.

The conclusion of our analysis is that the predicted magnetoresistance is very robust against a nonzero energy offset $\Delta$ and the presence of energetic disorder. Since the relevant dimensionless parameters are $\Delta / k_{\mathrm{B}} T$ and $\sigma / k_{\mathrm{B}} T$, this also means that the magnetoresistance is robust against a change in the temperature. We do note that a nonzero $\Delta$ or $\sigma$ creates energy barriers in the transport and that therefore the mobility itself strongly decreases with increasing $\Delta$ or $\sigma$.

\section{E. Influence of interchain hopping}

The huge magnetoresistance in doped polymers predicted in this work crucially depends on the effects of spin and charge blocking, which are only optimal for one-dimensional charge transport. However, in reality it might be difficult to separate individual chains far enough to completely prevent interchain hopping. We investigated the detrimental influence of

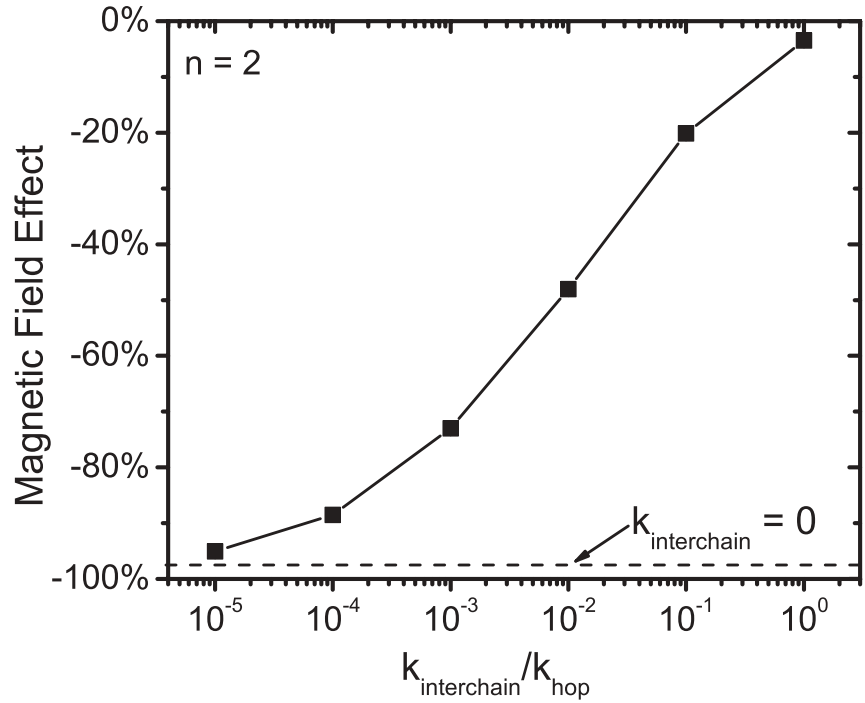

FIG. 8. (Color online) Magnetic field effect in the mobility as a function of the interchain hopping rate, $k_{\text {interchain }}$, for doping period $n=2$, energy offset $\Delta=0$, no disorder, and high electron density. The dashed line indicates the result for $k_{\text {interchain }}=0$.

interchain hopping on the magnetoresistance by MC simulations at high electron density, for $\Delta=0$ and no energetic disorder. In these simulations interchain hopping is modeled by allowing every electron to hop to a randomly chosen empty host site or to an ionized donor site within the chain with a rate $k_{\text {interchain }}$.

Figure 8 shows the magnetic field effect in the mobility as a function of $k_{\text {interchain }} / k_{\text {hop }}$ for doping period $n=2$. The size of the magnetic field effect decreases strongly with increasing $k_{\text {interchain }}$ to a very small value when $k_{\text {interchain }} / k_{\text {hop }}>$ 1. This result demonstrates that if the interchain hopping rate is not small enough, spin and charge blocking are not effective anymore, since electrons can hop to another chain when confronted with a spin-blocking or charge-blocking configuration.

\section{REALIZATION OF SUITABLE SYSTEMS}

We now come to the discussion of the possible realization of suitable systems that would show the predicted magnetoresistance. Two important conditions should be fulfilled: (1) The charges should be localized on monomers, with a hopping rate between monomers, $k_{\text {hop }}$, that is smaller than the hyperfine precession frequency, $\omega_{\mathrm{hf}}$; (2) Charge transport should be essentially one dimensional, which means that the interchain hopping rate must be much lower than the intrachain hopping rate.

Condition (1) could be fulfilled by inserting spacer units in between the monomers. We propose to use phenyl spacer units for this. It has been shown that with a single phenyl spacer the exchange coupling between a hole at a donor and an electron at an acceptor unit can be reduced to a value corresponding to a $\mathrm{mT}$, while with more phenyl spacers the coupling decreases exponentially with the number of phenyls. ${ }^{24}$ In the optimal case of vanishing $\Delta$, the condition that $k_{\text {hop }}$ is smaller than $\omega_{\text {hf }}$ means that the intrachain mobility (in the absence of an external magnetic field and for $n=2$ ) 
should not exceed $0.1 e \omega_{\mathrm{hf}} a^{2} / k_{\mathrm{B}} T$; see Fig. 3(a). With the typical values $\omega_{\mathrm{hf}}=10^{8} \mathrm{~s}^{-1}$ and $a=1 \mathrm{~nm}$ this leads to a maximal room-temperature mobility $\mu \approx 4 \times 10^{-6} \mathrm{~cm}^{2} / \mathrm{Vs}$. While this is not a very high mobility, the high charge density still leads to an appreciable current. Taking for the case $n=2$ half an electron per monomer unit with a volume of $1 \mathrm{~nm}^{3}$, such a mobility leads to a conductivity of about $0.3 \mathrm{~S} / \mathrm{cm}^{-1}$. This is not more than one order of magnitude lower than the conductivity of a conducting doped polymer like PEDOT:PSS [poly(3,4-ethylenedioxythiophene):poly(styrenesulfonate)].

Condition (2) could be fulfilled by adding side groups to the polymer such that polymer chains are far enough apart to prevent interchain hopping. Another interesting option might be blending with a nonconducting polymer. It has been shown that blends of poly(3-hexylthiophene) (P3HT) with nonconducting commodity polymers can show excellent conduction even at P3HT weight percentages of only a few percent. $^{25}$

A starting point for realizing the case $n=2$ could be the copolymerization of monomeric units with strongly electron accepting and electron donating properties. The onset of the optical transition for charge transfer in these polymers marks the energy needed to generate free charge carriers and has been made as low as $\Delta=0.5 \mathrm{eV}^{26,27}$ The mobility for these copolymers has been measured to be in the range $10^{-5}-10^{-3} \mathrm{~cm}^{2} / \mathrm{Vs},{ }^{27}$ which is too large to find a substantial magnetic field effect. Localization of the charges to the monomeric units and a sufficiently low hopping rate could be achieved by inserting spacer units in between the monomers, as discussed above.

Although a small value of the energy offset $\Delta$ is not needed to obtain a large magnetoresistance, it is an important condition for obtaining an appreciable mobility. It is interesting to note that the condition $\Delta \approx 0$ has been realized in molecularly doped organic semiconductors. An example is $\mathrm{F}_{4}$-TCNQ:ZnPc (tetrafluorotetracyanoquinodimethane:zinc phthalocyanine), which is used as hole-injection material in organic light-emitting diodes. ${ }^{28}$ In this system the LUMO energy of the acceptor ( $\left.\mathrm{F}_{4}-\mathrm{TCNQ}\right)$ and the HOMO energy of the host $(\mathrm{ZnPc})$ are nearly identical. The conduction in these systems is not one dimensional, so that the blocking effects discussed above will not be optimal. However, the existence of these systems shows that synthesis of $\pi$-conjugated organic units with $\Delta \approx 0$ should be possible.

We finally remark that a small magnetoresistance has been found in devices of PEDOT:PSS. ${ }^{29}$ It would be very interesting to investigate if the magnetoresistance in this polymer is of the type proposed in the present work. If this is the case, one could try to optimize the magnetoresistance along the route described in this paper.

\section{SUMMARY AND CONCLUSIONS}

In summary, we have investigated the magnetic field dependence of the charge mobility in a doped conjugated polymer by analytical and numerical methods. We considered the case of an electron donor in a host polymer and the limit that the electron hopping rate between the sites in the polymer is smaller than the hyperfine precession frequency of the electron spin. The largest magnetic-field effect in the mobility of $-98.5 \pm 0.3 \%$ was found for high doping concentration (equal amounts of donor and host sites) and high electron density (equal amounts of free electrons and ionized donors). The magnetic field effect arises because of the spin dependence of the recombination of an electron with an ionized donor (spin blocking) and the suppression of hyperfine-induced spin mixing by an external magnetic field. The increased effect at high electron density occurs because a single free electron-ionized donor pair in a spin-blocking configuration can block the current through the whole polymer chain (charge blocking). In addition, we found that energetic disorder and an imperfect alignment of the HOMO energy of the donor and the LUMO energy of the host polymer have only a minimal influence on the effect. The interchain hopping rate, however, does have a significant influence and should be low in order to obtain a large effect. We have suggested promising ways of realizing polymers that show the effect.

\section{ACKNOWLEDGMENT}

This work is part of the research program of the "Stichting voor Fundamenteel Onderzoek der Materie (FOM)," which is financially supported by the Nederlandse Organisatie voor Wetenschappelijk Onderzoek (NWO).
${ }^{1}$ J. Kalinowski, M. Cocchi, D. Virgili, P. D. Marco, and V. Fattori, Chem. Phys. Lett. 380, 710 (2003).

${ }^{2}$ T. L. Francis, O. Mermer, G. Veeraraghavan, and M. Wohlgenannt, New J. Phys. 6, 185 (2004).

${ }^{3}$ V. Prigodin, J. Bergeson, D. Lincoln, and A. Epstein, Synth. Met. 156, 757 (2006).

${ }^{4}$ P. Desai, P. Shakya, T. Kreouzis, and W. P. Gillin, Phys. Rev. B 76, 235202 (2007).

${ }^{5}$ B. Hu and Y. Wu, Nat. Mater. 6, 985 (2007).

${ }^{6}$ F. L. Bloom, W. Wagemans, M. Kemerink, and B. Koopmans, Phys. Rev. Lett. 99, 257201 (2007).

${ }^{7}$ F. J. Wang, H. Bässler, and Z. V. Vardeny, Phys. Rev. Lett. 101, 236805 (2008).
${ }^{8}$ U. Niedermeier, M. Vieth, R. Pätzold, W. Sarfert, and H. von Seggern, Appl. Phys. Lett. 92, 193309 (2008).

${ }^{9}$ T. D. Nguyen, G. Hukic-Markosian, F. Wang, L. Wojcik, X.-G. Li, E. Ehrenfreund, and Z. V. Vardeny, Nat. Mater. 9, 345 (2010).

${ }^{10}$ T. D. Nguyen, B. R. Gautam, E. Ehrenfreund, and Z. V. Vardeny, Phys. Rev. Lett. 105, 166804 (2010).

${ }^{11}$ U. E. Steiner and T. Ulrich, Chem. Rev. 89, 51 (1989).

${ }^{12}$ K. Schulten and P. Wolynes, J. Chem. Phys. 68, 3292 (1978).

${ }^{13}$ P. A. Bobbert, Nat. Mater. 9, 288 (2010).

${ }^{14}$ P. A. Bobbert, T. D. Nguyen, F. W. A. van Oost, B. Koopmans, and M. Wohlgenannt, Phys. Rev. Lett. 99, 216801 (2007). 
${ }^{15}$ S. P. Kersten, A. J. Schellekens, B. Koopmans, and P. A. Bobbert, Phys. Rev. Lett. 106, 197402 (2011).

${ }^{16}$ O. Mermer, G. Veeraraghavan, T. L. Francis, Y. Sheng, D. T. Nguyen, M. Wohlgenannt, A. Kohler, M. K. Al-Suti, and M. S. Khan, Phys. Rev. B 72, 205202 (2005).

${ }^{17}$ S. P. Kersten, A. J. Schellekens, B. Koopmans, and P. A. Bobbert, Synth. Met. 161, 613 (2011).

${ }^{18}$ A. J. Schellekens, W. Wagemans, S. P. Kersten, P. A. Bobbert, and B. Koopmans, Phys. Rev. B 84, 075204 (2011).

${ }^{19}$ V. Ambegaokar, B. I. Halperin, and J. S. Langer, Phys. Rev. B 4, 2612 (1971)

${ }^{20}$ W. F. Pasveer, J. Cottaar, C. Tanase, R. Coehoorn, P. A. Bobbert, P. W. M. Blom, D. M. de Leeuw, and M. A. J. Michels, Phys. Rev. Lett. 94, 206601 (2005).

${ }^{21}$ A. Miller and E. Abrahams, Phys. Rev. 120, 745 (1960).

${ }^{22}$ R. A. Marcus, Rev. Mod. Phys. 65, 599 (1993).
${ }^{23}$ S. Stafström, Chem. Soc. Rev. 39, 2484 (2010).

${ }^{24}$ Z. Dance, Q. Mi, D. McCamant, M. Ahrens, M. Ratner, and M. Wasielewski, Phys. Chem. B 110, 25163 (2006).

${ }^{25}$ S. Goffri, C. Müller, N. Stingelin-Stutzmann, D. W. Breiby, C. P. Radano, J. W. Andreasen, R. Thompson, R. A. J. Janssen, M. M. Nielsen, P. Smith, and H. Sirringhaus, Nat. Mater. 5, 950 (2006).

${ }^{26}$ E. Havinga, W. ten Hoeve, and H. Wynberg, Synth. Met. 55-57, 299 (1993).

${ }^{27}$ T. T. Steckler, X. Zhang, J. Hwang, R. Honeyager, S. Ohira, X.-H. Zhang, A. Grant, S. Ellinger, S. A. Odom, D. Sweat, D. B. Tanner, A. G. Rinzler, Stephen Barlow, J.-L. Brédas, B. Kippelen, S. R. Marder, and J. R. Reynolds, J. Am. Chem. Soc. 131, 2824 (2009).

${ }^{28}$ K. Walzer, B. Maennig, M. Pfeiffer, and K. Leo, Chem. Rev. 107, 1233 (2007).

${ }^{29}$ T. D. Nguyen, Y. Sheng, J. Rybicki, G. Veeraraghavan, and M. Wohlgenannt, J. Mater. Chem. 17, 1995 (2007). 